positive for S. haematobium, and a total of 132 (51\%) children developed 230 malaria attacks. Those with schistosomiasis had a $1.5: 1$ [1.1-2.0] risk to develop malaria compared to their uninfected counterparts. The mean number of malaria episodes per child over the study course was higher among children with schistosomiasis compared to those without (2.03 vs 1.57 , p-value $=0.015$ ).

Conclusion S. haematobium infection was associated with increased susceptibility to develop malaria (by increasing the risk to develop a malaria episode) and, consequently, a higher malaria incidence.

\section{PO 8458 POLYMORPHISM OF THE PLASMODIUM FALCIPARUM MSP-2 GENE ASSOCIATED WITH PLACENTAL MALARIA AT THE BORGOU-ALIBORI DEPARTMENTAL HOSPITAL}

Dossou Akpéyédjé*, Saadou Issifou. Fondation pour le Recherche Scientifique, Cotonou, Benin

\subsection{6/bmjgh-2019-EDC.104}

Background In sub-Saharan Africa, malaria during pregnancy is a major health problem because it poses significant risks for the pregnant woman and the foetus. The sequestration of Plasmodium falciparum-infected erythrocytes in the placenta has consequences for the mother and the foetus. This study aimed to evaluate the allelic polymorphism of the Plasmodium falciparum MSP-2 gene related to the consequences of placental malaria.

Methods It was a cross-sectional study conducted over two periods lasting six months in 2016 and 2017. The maternity center of the Hospital of Borgou-Alibori in Benin served as a framework for the study. From the 98 parturients included, placental blood samples were taken and then genotyped.

Results Using the MSP-2 gene as marker, the prevalence was 17, 34\%. The MSP-2 gene was polymorphic with 9 distinct allelic types for both 3D7 and FC27 families (150 bp; 200 bp; 250 bp; 275 bp; 300 bp; 350 bp; 400 bp; 450 bp and $500 \mathrm{bp}$ ). The FC27 allelic family was predominant over the $3 \mathrm{D} 7$ family with $56,25 \%$ and $43,75 \%$ respectively. The 300 bp allelic type (50\%) was predominant in the FC27 family while the $400 \mathrm{bp}$ type was predominant in 3D7 family $(35,71 \%)$. 9 women had polyclonality $(52,94 \%)$. The multiplicity of infection (MOI) was 1, 88. The number of strains ranged 1 to 4 in infected women. In univariate analysis there was no significant relationship between MSP-2 gene polymorphism and maternofoetal consequences. The absence of prenatal consultation $(p=0.0270)$, non-taking of IPTp/SP $(p=0.0060)$, the occurrence of malaria in the third trimester $(p=0,0364)$ and moderate maternal anaemia $(p=0.0277)$ were associated with the polymorphism of MSP-2 gene. The MOI was significantly associated with parasite density of infected women.

Conclusion Plasmodium falciparum MSP-2 gene was polymorphic in infected women at Parakou. Several factors related to pregnancy monitoring were associated with this genetic diversity. It is therefore essential to ensure correct follow-up of pregnancies.

\section{PO 8460 PANDORA-ID NET (PAN-AFRICAN NETWORK FOR RAPID RESEARCH, RESPONSE, RELIEF AND PREPAREDNESS FOR INFECTIOUS DISEASES EPIDEMICS)}

${ }^{1}$ Francine Ntoumi*, ${ }^{2}$ Francine Zumla, ${ }^{3}$ Giuseppe Ippolito, ${ }^{3}$ Francesco Vairo. ${ }^{1}$ Fondation Congolaise pour la Recherche Médicale, Brazzaville, Republic of the Congo; ${ }^{2}$ University College of London, London, UK; ${ }^{3}$ National Institute for Infectious Diseases Lazzaro Spallanzani, Rome, Italy

\subsection{6/bmjgh-2019-EDC.105}

Background New and re-emerging infectious disease outbreaks continue to cause much human suffering and loss of life worldwide. Since Africa has experienced repeated outbreaks of zoonotic infections, an important need exists to improve local and regional capacities to identify and respond to zoonotic outbreaks. PANDORA ID-NET is an EDCTP-supported 'ONE Human and Animal HEALTH' multidisciplinary consortium of 24 partner institutions (15 African and 9 European) in 9 African and 4 European countries.

Methods Our overall aim is to strengthen regional and panAfrican capacities and systems for enabling a rapid and effective response to infectious diseases with epidemic potential, arising from within Africa or imported from overseas. We aim to build laboratory and public health capabilites for rapid detection and surveillance of pathogens from human and animal sources. This will include obtaining accelerated evidence for optimal clinical management of patients, infection control measures, and public health response during outbreaks. Capacities will be built: a) for performing multisite clinical trials (evaluating rapid diagnostics, biomarkers, a range of treatments, vaccines and operational research studies) and, b) for timely collection, analysis and communication of information.

Conclusion Our activities will be aligned to EDCTP regional Networks of Excellence, Africa CDC and other relevant global and regional initiatives, thus maximizing complementarity and achieving a multiplier effect, facilitating rapid policy implementation of outputs.

\section{PO 8467 IMPACT OF COMMUNITY CASE MANAGEMENT OF MALARIA (CCMM) AMONG CHILDREN UNDER FIVE YEARS OLD: AN EVALUATIVE STUDY IN KABONGA}

${ }^{1}$ Ciza Bonne*, ${ }^{2}$ Kagozi Husseni, ${ }^{3}$ Ciza Josephine Muhigirwa. ${ }^{1}$ Health Healing Network Burundi, Bujumbura, Burundi; ${ }^{2}$ Solidarity of nurses for promotion of maternal and children's health, Burundi; ${ }^{3}$ Palliative care association of Burundi, Bujumbura, Burundi

\subsection{6/bmjgh-2019-EDC.106}

Background In Burundi, malaria is considered a major public health concern and the leading cause of death. Malaria is responsible for up to $25 \%$ of all outpatient visits and up to $48 \%$ of all deaths in health facilities among children under five years old. Despite efforts made, timely access to health care is still limited, mainly due to geographic inaccessibility and lack of awareness about malaria complications. To increase timely access to malaria treatment, Burundi's Ministry of Health implemented Community Case Management of Malaria (CCMM) since 2015 in Kabonga. We sought to assess the impact of CCMM in improving malaria outcomes following two years of its initiation. 